\title{
Predictive value of d-dimer/albumin ratio and fibrinogen/albumin ratio for in-hospital mortality in patients with COVID-19
}

\author{
Kadir Küçükceran ${ }^{1}$, Mustafa Kürşat Ayranc1 $^{1}$, Sadik Girisgin $^{1}$, and Sedat Koçak ${ }^{1}$ \\ ${ }^{1}$ Necmettin Erbakan University Meram Medical Faculty Hospital
}

April 3, 2021

\begin{abstract}
Introduction: Due to the high mortality of coronavirus disease 2019 (COVID-19), there are difficulties in the managing emergency department. We investigated whether the d-dimer/albumin ratio (DAR) and fibrinogen/albumin ratio (FAR) predicts mortality in the COVID-19 patients.

Methods: A total of 717 COVID-19 patients who were brought to the emergency department from March to October 2020 were included in the study. Levels of d-dimer, fibrinogen, and albumin, as well as DAR, FAR, age, gender, and in-hospital mortality status of the patients were recorded. The patients were grouped by in-hospital mortality. Statistical comparison was conducted between the groups.

Results: Of the patients included in the study, 371(51.7\%) were male, and their median age was 64 years (50-74). There was in-hospital mortality in $126(17.6 \%)$ patients. The area-under-the-curve (AUC) and odds ratio values obtained by DAR to predict in-hospital mortality were higher than the values obtained by the all other parameters (AUC of DAR, albumin, d-dimer, FAR, and fibrinogen: 0.773, 0.766, 0.757, 0.703, and 0.637, respectively; odds ratio of DAR $>56.36$, albumin $<4.015$, d-dimer $>292.5$, FAR $>112.33$, and fibrinogen $>423: 7.898,6.216,6.058,4.437$, and 2.794, respectively). In addition; patients with concurrent DAR $>56.36$ and FAR $>112.33$ had an odds ratio of 21.879 with respect to patients with concurrent DAR $<56.36$ and FAR $<112.33$
\end{abstract}

Conclusion: DAR may be used as a new marker to predict mortality in COVID-19 patients. In addition the concurrent high DARs and FARs were found to be more valuable in predicting in-hospital mortality than either separately.

Keywords: Covid-19, D-dimer, Fibrinogen, Serum Albumin, in-Hospital Mortality

Introduction: Due to the high mortality of coronavirus disease 2019 (COVID-19), there are difficulties in the managing emergency department. We investigated whether the d-dimer/albumin ratio (DAR) and fibrinogen/albumin ratio (FAR) predicts mortality in the COVID-19 patients.

Methods: A total of 717 COVID-19 patients who were brought to the emergency department from March to October 2020 were included in the study. Levels of d-dimer, fibrinogen, and albumin, as well as DAR, FAR, age, gender, and in-hospital mortality status of the patients were recorded. The patients were grouped by in-hospital mortality. Statistical comparison was conducted between the groups.

Results: Of the patients included in the study, 371(51.7\%) were male, and their median age was 64 years (5074). There was in-hospital mortality in $126(17.6 \%)$ patients. The area-under-the-curve (AUC) and odds ratio values obtained by DAR to predict in-hospital mortality were higher than the values obtained by the all other parameters (AUC of DAR, albumin, d-dimer, FAR, and fibrinogen: 0.773, 0.766, 0.757, 0.703, and 0.637, respectively; odds ratio of $\mathrm{DAR}>56.36$, albumin $<4.015$, d-dimer $>292.5, \mathrm{FAR}>112.33$, and fibrinogen $>423$ : $7.898,6.216,6.058,4.437$, and 2.794 , respectively). In addition; patients with concurrent DAR $>56.36$ and FAR $>112.33$ had an odds ratio of 21.879 with respect to patients with concurrent $\mathrm{DAR}<56.36$ and FAR $<112.33$.

Conclusion: DAR may be used as a new marker to predict mortality in COVID-19 patients. In addition the concurrent high DARs and FARs were found to be more valuable in predicting in-hospital mortality than 
either separately.

Keywords: Covid-19, D-dimer, Fibrinogen, Serum Albumin, in-Hospital Mortality

Predictive value of $\mathrm{d}$-dimer/albumin ratio and fibrinogen/albumin ratio for in-hospital mortality in patients with COVID-19

Kadir KÜÇÜKCERAN ${ }^{1}$ kadirkucukceran@hotmail.com0000-0001-9758-0803

Mustafa Kürşat AYRANCI ${ }^{1}$ drmka157@gmail.com0000-0002-7196-0856

Abdullah Sadık GİİŞGiN ${ }^{1}$ sgirisgin@yahoo.com0000-0003-2669-0532

Sedat KOÇAK ${ }^{1}$ drskocak06@hotmail.com0000-0001-8265-9615

${ }^{1}$ Emergency Department, Necmettin Erbakan University, Meram School of Medicine, Konya

Running Title: Role of DAR in predicting mortality

Keywords : Covid-19, D-dimer, Fibrinogen, Serum Albumin, in-Hospital Mortality

Ethics Committee Approve: Necmettin Erbakan University Meram Medical Faculty Pharmaceutical and Non-Medical Device Studies Ethical Committee by the decision number of 2021/3022

Funding: This study did not need financial funding

Conflict of Interest: The authors declare no conflict of interest

Human rights: During the research, the World Medical Association(WMA) Declaration of HELSINKI and/ or the World Psychiatric Association HAWAII Declaration of Good Clinical Practice rules were complied with

Corresponding author: Dr. Kadir Küçükceran

Necmettin Erbakan University, Meram School of Medicine, Konya, Turkey

Phone: +905055532414/+903322234053

e-mail:kadirkucukceran@hotmail.com

\section{ABSTRACT}

Introduction: Due to the high mortality of coronavirus disease 2019 (COVID-19), there are difficulties in the managing emergency department. We investigated whether the d-dimer/albumin ratio (DAR) and fibrinogen/albumin ratio (FAR) predicts mortality in the COVID-19 patients.

Methods: A total of 717 COVID-19 patients who were brought to the emergency department from March to October 2020 were included in the study. Levels of d-dimer, fibrinogen, and albumin, as well as DAR, FAR, age, gender, and in-hospital mortality status of the patients were recorded. The patients were grouped by in-hospital mortality. Statistical comparison was conducted between the groups.

Results: Of the patients included in the study, 371(51.7\%) were male, and their median age was 64 years (50-74). There was in-hospital mortality in $126(17.6 \%)$ patients. The area-under-the-curve (AUC) and odds ratio values obtained by DAR to predict in-hospital mortality were higher than the values obtained by the all other parameters (AUC of DAR, albumin, d-dimer, FAR, and fibrinogen: 0.773, 0.766, 0.757, 0.703 , and 0.637 , respectively; odds ratio of $\mathrm{DAR}>56.36$, albumin $<4.015$, d-dimer $>292.5$, FAR $>112.33$, and fibrinogen $>423: 7.898,6.216,6.058,4.437$, and 2.794, respectively). In addition; patients with concurrent $\mathrm{DAR}>56.36$ and $\mathrm{FAR}>112.33$ had an odds ratio of 21.879 with respect to patients with concurrent $\mathrm{DAR}<56.36$ and $\mathrm{FAR}<112.33$. 
Conclusion: DAR may be used as a new marker to predict mortality in COVID-19 patients. In addition the concurrent high DARs and FARs were found to be more valuable in predicting in-hospital mortality than either separately.

Keywords: Covid-19, D-dimer, Fibrinogen, Serum Albumin, in-Hospital Mortality

\section{Introduction}

In December 2019, a disease of unknown etiology emerged in Wuhan, China, with clinical symptoms of acute upper respiratory tract infection (1). This disease, called coronovirus disease 2019 (COVID-19), was evaluated in the pandemic category by the World Health Organization (WHO) in January 2020 (2). According to WHO data, approximately 117 million people have been infected, while approximately 2.6 million people died. The mortality rate is $30 \%$ in hospitalized cases (3), ranging between $30 \%$ and $100 \%$ in cases requiring a ventilator (4). Hospitalized COVID-19 patients pose difficulties in emergency department management, especially due to high mortality rates. In order to accelerate the outcome of COVID 19 patients in the emergency department, parameters to predict mortality that can be applied easily and quickly are needed.

Hypercoagulation, which is the cause of high d-dimer and fibrinogen, is common in hospitalized COVID-19 patients, and is a predictor of severity (5). Fibrinogen to albumin ratio (FAR) was found to be more sensitive and specific predictor than fibrinogen in showing the progression of hypercoagulation (6). Some studies of COVID 19 patients have used d-dimer, albumin, fibrinogen and FAR for this purpose (7-9), but to the best of our knowledge, no studies have used the d-dimer to albumin ratio (DAR). Therefore, in this study we aimed to investigate and compare the predictive value of DAR and FAR for mortality in patients with COVID-19.

\section{Method}

Ethics committee approval for this single-center, retrospective, and observational study was obtained from the Necmettin Erbakan University Meram Medical Faculty Pharmaceutical and Non-Medical Device Studies Ethical Committee, decision number of 2021/3022.

This study was carried out in a tertiary university hospital. Patients suspected of having COVID-19 who were admitted to the emergency department and hospitalized and whose PCR test was positive were included in the study from March to October 2020. Regardless of the number of tests submitted, any PCR result that appeared positive at least once was considered positive, while other results were considered negative. Patients who were discharged against medical advice and referred to other hospitals were excluded from the study. Patients, who were discharged against medical advice; transferred to other hospitals and had no results of at least one of the d-dimer, fibrinogen and albumin parameters were excluded.

The following pieces of patient data were recorded from the patients' e-files using the Hospital Information Management Systems program: d-dimer level; fibrinogen level; albumin level; complaint (fever, cough, shortness of breath); vital signs; comorbidities; information about their ward/ICU admission; hospital outcome (discharge, exitus in-hospital); and in-hospital mortality status. The DAR was obtained by dividing the d-dimer level by the albumin level. The FAR was obtained by dividing the fibrinogen level by the albumin level. The primary outcomes of the study were prediction of in-hospital mortality using DAR and FAR and determination of whether DAR or FAR was a more accurate predictor than using d-dimer, fibrinogen, and albumin levels.

Statistical analysis of the data was performed using the SPSS 20.0 (SPSS Inc., Chicago, IL) package program. Histograms and the Kolmogorov-Smirnov test was used to test the normality of the data. Following the normality analysis result, all quantitative data were expressed as median ( $25 \%-75 \%$ quarters) since they did not show a normal distribution, while categorical variables were expressed as frequency (percentage). The differences between the groups were investigated using the Mann-Whitney U test. Intragroup comparisons of the categorical variables were made using the chi square test and the Fisher's exact test. Receiver operating characteristic (ROC) analysis was performed to determine predictive power of the d-dimer, fibrinogen, and albumin levels and DAR and FAR for in-hospital mortality. The optimum cut-off levels of the biochemical parameters were determined using Youden's index (sensitivity +1 -specificity). The sensitivity, specificity, 
and positive and negative predictive values of the parameters were calculated for the optimum cut-off levels. The odds ratios of the groups categorized by the optimum cut-off values of d-dimer, fibrinogen, albumin, DAR, and FAR in predicting in-hospital COVID-19 mortality were calculated using univariate logistic regression analysis. The area under the curve (AUC) and odds ratio values were used to compare the mortality predictive power of parameters. Statistical significance was set at $\mathrm{p}<0.05$.

\section{Results}

A total of 749 patients who were brought to the emergency department with suspected COVID-19 within the period from March to September 2020 and who had a positive PCR test result were identified. Of these 749 patients, 11 were excluded from the study because they sought hospital discharge against medical advice, 8 because they were referred to another hospital, 13 because they had no result of at least one of the d-dimer, fibrinogen and albumin parameters. The remaining 717 patients were included in the study.

Of the 717 patients who were included in the study, 371(51.7\%) were male, their median age was 64 years (50-74), and their median length of hospital stay was 9 days (5-14). The median d-dimer, fibrinogen, albumin, DAR, and FAR values were found to be $223(130-454) \mathrm{ng} / \mathrm{mL}, 419(324-534) \mathrm{mg} / \mathrm{dL}, 4.05(3.68-$ $4.42) \mathrm{g} / \mathrm{dL}, 56(30.43-119.69)$ and 104.3(77.46-138.88), respectively. A total of 495 patients (69\%) had at least one comorbidity in their medical history, the most frequent which was hypertension (260 patients, $36.3 \%)$. A total of 550 patients $(76.7 \%)$ were hospitalized in the ward unit, and $167(23.3 \%)$ were admitted to the ICU. In-hospital mortality occurred in 126 patients (17.6\%) and did not occur in 591(76.7\%). Table 1 shows the detailed data of the cases.

The median d-dimer value in the non-survivor group was significantly higher than that in the survivor group (non-survivor: $471 \mathrm{ng} / \mathrm{mL}$ [264-869]; survivor: $184 \mathrm{mg} / \mathrm{dL}$ [116.5-364]; $\mathrm{p}<0.001$ ). The median fibrinogen value, that in the non-survivor group was significantly higher than in the survivor group (non-survivor: $474 \mathrm{mg} / \mathrm{dL}$ [388-577]; survivor: $403 \mathrm{mg} / \mathrm{dL}$ [315-514.5]; $\mathrm{p}<0.001$ ). The median albumin value, that in the non-survivor group was significantly lower than that in the survivor group (non-survivor: $3.6 \mathrm{~g} / \mathrm{dL}$ [3.31-3.95]; survivor: $4.16 \mathrm{~g} / \mathrm{dL}$ [3.79-4.48]; $\mathrm{p}<0.001)$. The median DAR value in the non-survivor group was significantly higher than that in the survivor group (non-survivor: 127.79 [75.5-240.2]; survivor: 47.8 [27.29-94.47]; $\mathrm{p}<0.001)$. The median FAR value in the non-survivor group was significantly higher than in the survivor group (non-survivor: 133.49 [108.27-161.18]; survivor: 98.12 [73.56-131.84]; $\mathrm{p}<0.001$ ). Table 2 shows the detailed intragroup comparisons by in-hospital mortality.

We performed ROC analysis to determine the in-hospital COVID-19 mortality predictive power of the ddimer, fibrinogen, and albumin levels, and DAR and FAR. The AUC value of the DAR was found to be 0.773 (Figure 1), and $84.9 \%$ sensitivity, $58.4 \%$ specificity, $30.3 \%$ PPD, and $94.8 \%$ NPD were reached with a DAR cut-off value of 56.36. For FAR, its AUC value was found to be 0.703 (Figure 1), and $71.4 \%$ sensitivity, $64 \%$ specificity, $29.7 \%$ PPD, and $91.3 \%$ NPD were reached with a FAR cut-off value of 112.33 . The detailed results of the ROC analysis are given in Table 3.

The odds ratios for predicting in-hospital COVID-19 mortality were as follows: patients with d-dimer $>292.5$ $\mathrm{ng} / \mathrm{mL}$ had an odds ratio of 6.058 , those with fibrinogen $>423 \mathrm{mg} / \mathrm{dL}$ had 2.794 , those with albumin $<4.015$ $\mathrm{g} / \mathrm{dL}$ had 6.216, those with DAR $>56.36$ had 7.898, and those with FAR $>112.33$ had 4.437. In addition, patients with concurrent DAR $>56.36$ and FAR $>112.33$ had an odds ratio of 21.879 with respect to patients with concurrent DAR $<56.36$ and FAR $<112.33$. Detailed odds ratio results are given in Table 4.

\section{Discussion}

In this study, we investigated d-dimer, fibrinogen, albumin, DAR, and FAR parameters as predictors for in-hospital mortality of COVID-19 patients. According to the results of this study, while DAR was found to be most valuable predictive parameters, FAR was found to be more valuable as a predictor than only fibrinogen. In addition concurrent high DAR and FAR values as compared to concurrent low DAR and FAR values were found to be more predictively valuable than high values of DAR and FAR separately with respect to low DAR and FAR taken separately. 
According to the results of the ROC analysis performed to predict in-hospital mortality, the d-dimer levels reached a 0.757 AUC value and d-dimer levels above $292.5 \mathrm{ng} / \mathrm{mL}$ had a predictive odds ratio of 6.058. Other studies have also associated high d-dimer levels with high mortality. Zhang et al. (10) carried out a study with 343 COVID-19 patients and found that a d-dimer level with a 0.89 AUC value was able to predict in-hospital mortality. Zhou et al. (11) studied a total of 191 COVID-19 patients with 54 patients in the nonsurvivor group and found that a d-dimer level above $1 \mu \mathrm{g} / \mathrm{mL}$ reached an odds ratio of 20.04 in predicting mortality with respect to a d-dimer level below $0.5 \mu \mathrm{g} / \mathrm{mL}$.

There are many possible reasons for the relationship between a high d-dimer level and COVID-19 mortality. The fact that COVID-19 causes hypercoagulation (12), may explain this relationship, because it can increase the risk of microthrombus formation and further aggravate the risk of organ failure due to inflammation (13). High d-dimer levels are expected as a result of hypercoagulation (14). Pulmonary embolism and venous thromboembolism are more frequent in hospitalized COVID-19 patients compared to patients hospitalized for other reasons (5). Pulmonary intravascular coagulation and vascular occlusion are frequent findings reported in autopsies of patients who died of COVID-19 (5). COVID-19 leads to an increased risk of arterial thrombosis such as ischemic stroke, myocardial infarction and limb ischemia (5). Hypercoagulation due to COVID-19 may occur for various reasons. Hypoxia caused by COVID-19 triggering thrombosis may be one of these reasons (15). In addition, patients with severe COVID-19 are more comorbid, more immobile, and exposed to more invasive procedures, increasing the likelihood of thrombotic events (10).

The results of the ROC analysis performed to predict in-hospital mortality showed that fibrinogen levels reached a 0.637 AUC value. Fibrinogen levels above $423 \mathrm{mg} / \mathrm{dL}$ had an odds ratio of 2.794 in predicting the in-hospital mortality of COVID-19 patients. Bi et al. (9) found that fibrinogen levels above $4 \mathrm{~g} / \mathrm{L}$ reached a hazard ratio of 3.284 in predicting disease severity with respect to fibrinogen levels below $4 \mathrm{~g} / \mathrm{L}$. The reason for the relationship between high fibrinogen levels and COVID-19 mortality is hypercoagulation (16), which is also caused by high d-dimer levels. Hyperfibrinogenemia increases the risk of coronary artery disease, deep vein thrombosis, and ischemic stroke (17-19). Although both d-dimer and fibrinogen are indicators of coagulation and inflammation, d-dimer was found to be more predictive of in-hospital mortality than fibrinogen (AUC of d-dimer and fibrinogen: 0.757, 0.637; odds ratio of d-dimer and fibrinogen: 6.058, 2.794). The reason of this is that while fibrinogen levels increase in the early stage of inflammation, they tend to peak and then decrease in the late stages when the disease is severe (16).

In the ROC analysis performed to predict in-hospital mortality, albumin levels reached a 0.766 AUC value. Albumin levels below $4.015 \mathrm{~g} / \mathrm{dL}$ had an odds ratio of 6.216 . This is consistent with the results of prior studies. For example, de la Rica et al. (20) found that in a total of 48 COVID-19 patients with 21 patients in the ICU group, albumin levels were statistically significantly lower in ICU group than non-ICU group. Violi et al. (8) found that in a total of 319 hospitalized COVID-19 patients with 64 patients in the nonsurvivor group, albumin levels below $3.2 \mathrm{~g} / \mathrm{dL}$ had an odds ratio of 2.48 in predicting in-hospital mortality. As with d-dimer and fibrinogen, hypercoagulation is one possible explanation for the relationship between hypoalbuminemia and COVID-19 mortality. Some studies have shown that arterial and venous thromboembolic events increase with hypoalbuminemia $(21,22)$.

Finally, according to the result of the ROC analysis performed to predict in-hospital COVID-19 mortality, the DAR and FAR AUCs reached 0.773 and 0.703 , respectively. While DAR $>56.36$ had an odds ratio of 7.898 and FAR $>112.33$ had an odds ratio of 4.437. Some studies have indicated that FARs increase in hypercoagulation states such as cardiovascular disease, cerebrovascular disease, and chronic venous insufficiency $(6,23,24)$. As mentioned earlier, we did not any prior studies of the predictive value of DAR. The study Bi et al. (9) of a total of 113 COVID-19 patients with 22 patients in the serious group found that hazard ratios obtained by FAR for predicting severity of COVID-19 were higher than those obtained by fibrinogen levels (hazard ratio of FAR >0.0883: 5.212, hazard ratio of fibrinogen >4: 3.284). In addition, Karahan et al. (6) found FAR to be a more sensitive and specific predictor than fibrinogen in showing the progression of venous insufficiency. In this study, the AUC and odds ratio values obtained using FARs for predict in-hospital COVID-19 mortality were higher than the values obtained by only using fibrinogen levels. However, the AUC and odds ratio values 
obtained using DARs to predict in-hospital COVID-19 mortality were higher than the values obtained using any other parameter. In addition; we found that in-hospital mortality risk due to concurrent high DARs and FARs compared to concurrent low DARs and FARs (odds ratio: 21.879) were found to be higher than the in-hospital mortality risk when evaluated separately (DAR odds ratio: 7.898, FAR odds ratio: 4.437). We have not found any previous studies in the literature evaluating concurrent high levels of DAR and FAR.

The limitations of our study were as follows: the limited number of cases, being a retrospective and singlecenter study, exclusion of discharged COVID-19 patients in the study, and failure to evaluate treatment protocols. Another limitation is that patients using anticoagulant drugs were not excluded from the study; patients using anticoagulant drugs may be less affected by hypercoagulation due to COVID-19.

\section{Conclusion}

In this study while FAR was found to be more valuable than using only fibrinogen levels to predict in-hospital COVID-19 mortality, DAR was found to be more valuable than any other parameter. In addition, concurrent high DAR and FAR were found to be more predictive of in-hospital COVID-19 mortality than either taken separately.

\section{References}

1. Yang X, Yu Y, Xu J, Shu H, Liu H, Wu Y, et al. Clinical course and outcomes of critically ill patients with SARS-CoV-2 pneumonia in Wuhan, China: a single-centered, retrospective, observational study. Lancet Respir Med. 2020;8(5):475-81.

2. Demir ET, Kilic F. Determination of The Anxiety Level in Pregnant Women Who Administer to The Obstetrics Clinic Within The Covid-19 Pandemia Period. Selcuk Med J 2020;36(4):352-356.

3. Martins-Filho PR, de Souza Araújo AA, Pereira LX, Quintans-Júnior LJ, de Souza Barboza W, Cavalcante TF, et al. Factors Associated with Mortality among Hospitalized Patients with COVID-19: A Retrospective Cohort Study. Am J Trop Med Hyg. 2020;104(1):103-5.

4. Akbudak İ. Retrospective evaluation of critical patients followed in intensive care with the diagnosis of Covid-19 infection: single center experience. Pam Med J. 2021; 14(2): 18-18.

5. Gerotziafas GT, Catalano M, Colgan M-P, Pecsvarady Z, Wautrecht JC, Fazeli B, et al. Guidance for the management of patients with vascular disease or cardiovascular risk factors and COVID-19: position paper from VAS-European Independent Foundation in Angiology/Vascular Medicine. Thromb Haemost. 2020;120(12):1597.

6. Karahan O, Yavuz C, Kankilic N, Demirtas S, Tezcan O, Caliskan A, et al. Simple blood tests as predictive markers of disease severity and clinical condition in patients with venous insufficiency. Blood Coagul Fibrinolysis. 2016;27(6):684-90.

7. Bansal A, Singh AD, Jain V, Aggarwal M, Gupta S, Padappayil RP, et al. A Systematic Review and Meta-analysis of D-Dimer Levels in Patients Hospitalized with Coronavirus Disease 2019 (COVID-19). medRxiv. 2020

8. Violi F, Cangemi R, Romiti GF, Ceccarelli G, Oliva A, Alessandri F, et al. Is albumin predictor of mortality in COVID-19? Antioxidants \& redox signaling. 2020.

9. Bi X, Su Z, Yan H, Du J, Wang J, Chen L, et al. Prediction of severe illness due to COVID-19 based on an analysis of initial Fibrinogen to Albumin Ratio and Platelet count. Platelets. 2020;31(5):674-9.

10. Zhang L, Yan X, Fan Q, Liu H, Liu X, Liu Z, et al. D-dimer levels on admission to predict in-hospital mortality in patients with Covid-19. J Thromb Haemost. 2020;18(6):1324-9.

11. Zhou F, Yu T, Du R, Fan G, Liu Y, Liu Z, et al. Clinical course and risk factors for mortality of adult inpatients with COVID-19 in Wuhan, China: a retrospective cohort study. The lancet. 2020;395(10229):10541062. 
12. Connors JM, Levy JH. Thromboinflammation and the hypercoagulability of COVID-19. Thromb Haemost. 2020;18(7):1559-61.

13. So LK, Lau AC, Yam LY, Cheung TM, Poon E, Yung RW, et al. Development of a standard treatment protocol for severe acute respiratory syndrome. The Lancet. 2003;361(9369):1615-7.

14. Danese E, Montagnana M, Cervellin G, Lippi G. Hypercoagulability, D-dimer and atrial fibrillation: an overview of biological and clinical evidence. Ann Med. 2014;46(6):364-71.

15. Gupta N, Zhao Y-Y, Evans CE. The stimulation of thrombosis by hypoxia. Thromb Res. 2019;181:7783.

16. Thachil J. The protective rather than prothrombotic fibrinogen in COVID-19 and other inflammatory states. J Thromb Haemost. 2020;18(8):1849-52.

17. Karahan O, Acet H, Ertaş F, Tezcan O, Çalişkan A, Demir M, et al. The relationship between fibrinogen to albumin ratio and severity of coronary artery disease in patients with STEMI. Am J Emerg Med. 2016;34(6):1037-42.

18. Wuillemin W, Korte W, Waser G, Lämmle B. Usefulness of the D-dimer/fibrinogen ratio to predict deep venous thrombosis. J Thromb Haemost. 2005;3(2):385-7.

19. Shi J, Shi R, Qin W, Zhang X, Liu H, Xu J, et al. Dynamic Changes in Fibrinogen and Prognosis of Acute Ischemic Stroke Patients Treated with Intravenous Thrombolysis. Neurotox Res. 2020;38(3):775-84.

20. de la Rica R, Borges M, Aranda M, Del Castillo A, Socias A, Payeras A, et al. Low albumin levels are associated with poorer outcomes in a case series of COVID-19 patients in Spain: a retrospective cohort study. Microorganisms. 2020;8(8):1106.

21. Chi G, Gibson CM, Liu Y, Hernandez AF, Hull RD, Cohen AT, et al. Inverse relationship of serum albumin to the risk of venous thromboembolism among acutely ill hospitalized patients: Analysis from the APEX trial. Am J Hematol. 2019;94(1):21-8.

22. Ronit A, Kirkegaard-Klitbo DM, Dohlmann TL, Lundgren J, Sabin CA, Phillips AN, et al. Plasma albumin and incident cardiovascular disease: results from the CGPS and an updated meta-analysis. Arterioscler Thromb Vasc Biol. 2020;40(2):473-82.

23. Sapmaz I, Saba T, Haberal C, Toktamis A, Cakmak M, Cicek D. Fibrinogen-albumin ratio: an intriguing relationship for assessing trombosis risk and suspicious effect on blood viscosity. International Cardiovascular Research Journal. 2011; 5(4):153-154.

Ozcan Cetin EH, Konte HC, Temizhan A. Blood viscosity should not be overlooked when evaluating the fibrinogen to albumin ratio. Angiology. 2019;70(5):465-6.

1.

Table 1: Features of Participants

\begin{tabular}{lll}
\hline Number of Persons & & $717(100 \%)$ \\
\hline Age & & $64(50-74)$ \\
Gender & Male & $371(51.7 \%)$ \\
& Female & $346(48.3 \%)$ \\
Vital Signs & Fever (C $)$ & $36.5(36.2-36.9)$ \\
& Pulse (per minute) & $91(82-103)$ \\
& Systolic Blood Pressure (mmHg) & $130(120-141)$ \\
& Diastolic Blood Pressure (mmHg) & $74(70-80)$
\end{tabular}




\begin{tabular}{lll}
\hline Number of Persons & & $717(100 \%)$ \\
\hline & MAP (mmHg) & $93(86.66-101)$ \\
Complaints & Saturation (\%) & $93(90-96)$ \\
& Fever & $357(49.8 \%)$ \\
Laboratory Results & Cough & $376(52.4 \%)$ \\
& Shortness of Breath & $343(47.8 \%)$ \\
& D-Dimer $(\mathrm{ng} / \mathrm{mL})$ & $223(130-454)$ \\
& Fibrinojen (mg/dL) & $419(324-534)$ \\
& Albumin $(\mathrm{g} / \mathrm{dL})$ & $4.05(3.68-4.42)$ \\
Medical History & DAR & $56(30.43-119.69)$ \\
& FAR & $104.3(77.46-138.88)$ \\
& Comorbidity & $495(69 \%)$ \\
& Hypertension & $260(36.3 \%)$ \\
& Diabetes Mellitus & $197(27.5 \%)$ \\
& Cardiovascular Disease & $137(19.1 \%)$ \\
& Asthma-COPD & $118(16.5 \%)$ \\
Length of Hospital Stay (Day) & Malignancy & $74(10.3 \%)$ \\
Emergency Service Outcome & CRF & $31(4.3 \%)$ \\
& Ward Unit & $16(2.2 \%)$ \\
Hospital Outcome & ICU & $9(5-14)$ \\
In-Hospital Mortality & Discharged & $550(76.7 \%)$ \\
& Ex & $167(23.3 \%)$ \\
& Survivor & $591(76.7 \%)$ \\
& Nonsurvivor & $126(17.6 \%)$ \\
\hline
\end{tabular}

COPD: Chronic Obstructive Pulmonary Disease, MAP: Mean Arterial Pressure, ICU: Intensive Care Unit, CRF: Chronic Renal Failure

* Having at least one additional disease in his / her medical history

Table 2: Evaluation of Participants by In-Hospital Mortality

\begin{tabular}{|c|c|c|c|c|}
\hline & & Non-survivor(126) & Survivor(591) & $\mathrm{p}$ value \\
\hline Age & Age & $76(68-83)$ & $61(46.5-71)$ & $<0.001$ \\
\hline Temperature $\left({ }^{\circ} \mathrm{C}\right)$ & Temperature $\left({ }^{\circ} \mathrm{C}\right)$ & $36.7(36.3-36.7)$ & $36.5(36.1-36.9)$ & 0.029 \\
\hline Pulse (per minute) & Pulse (per minute) & $90(81-106)$ & $92(82-103)$ & 0.715 \\
\hline SBP (mmHg) & $\mathrm{SBP}(\mathrm{mmHg})$ & 130(110-144) & 130(120-140) & 0.165 \\
\hline DBP (mmHg) & DBP (mmHg) & $70(60-80$ & $75(70-80)$ & 0.001 \\
\hline MAP (mmHg) & MAP (mmHg) & $90(79-100)$ & $93(87-101.33)$ & 0.009 \\
\hline Saturation (\%) & Saturation (\%) & $89(80-93)$ & $94(91-96)$ & $<0.001$ \\
\hline D-dimer $(\mathrm{ng} / \mathrm{mL})$ & D-dimer $(\mathrm{ng} / \mathrm{ml})$ & $471(264-869)$ & $184(116.5-364)$ & $<0.001$ \\
\hline Fibrinogen $(\mathrm{mg} / \mathrm{dL})$ & Fibrinogen (mg/dl) & $474(388-577)$ & $403(315-514.5)$ & $<0.001$ \\
\hline Albumin $(\mathrm{g} / \mathrm{dL})$ & $\operatorname{Albumin}(\mathrm{g} / \mathrm{dl})$ & $3.6(3.31-3.95)$ & $4.16(3.79-4.48)$ & $<0.001$ \\
\hline DAR & DAR & $127.79(75.5-240.2)$ & $47.8(27.29-94.47)$ & $<0.001$ \\
\hline FAR & FAR & $133.49(108.27-161.18)$ & $98.12(73.56-131.84)$ & $<0.001$ \\
\hline Length of hospital stay (Days) & Length of hospital stay (Days) & $13(9-23)$ & $8(5-12)$ & $<0.001$ \\
\hline \multirow[t]{2}{*}{ Gender } & Male & $78(61.9 \%)$ & $293(49.6 \%)$ & 0.012 \\
\hline & Female & $48(38.1 \%)$ & $298(50.4 \%)$ & \\
\hline Fever & Fever & $52(41.3 \%)$ & $305(51.6 \%)$ & 0.035 \\
\hline
\end{tabular}




\begin{tabular}{lllll}
\hline & & Non-survivor(126) & Survivor(591) & p value \\
\hline Cough & Cough & $66(52.4 \%)$ & $310(52.5 \%)$ & 0.988 \\
Shortness of Breath $^{*}$ & Shortness of Breath $^{*}$ & $83(65.9 \%)$ & $260(44 \%)$ & $<0.001$ \\
Comorbidity & Comorbidity $^{*}$ & $108(85.7 \%)$ & $387(65.5 \%)$ & $<0.001$ \\
Hypertension & Hypertension & $64(50.8 \%)$ & $196(33.2 \%)$ & $<0.001$ \\
Diabetes Mellitus & Diabetes Mellitus & $42(33.3 \%)$ & $155(26.2 \%)$ & 0.105 \\
Asthma-COPD & Asthma-COPD & $20(15.9 \%)$ & $98(16.6 \%)$ & 0.845 \\
Cardiovascular Disease & Cardiovascular Disease & $35(27.8 \%)$ & $102(17.3 \%)$ & 0.006 \\
Malignancy & Malignancy & $21(16.7 \%)$ & $53(9 \%)$ & 0.010 \\
CRF & CRF & $11(8.7 \%)$ & $20(3.4 \%)$ & 0.007 \\
Cerebrovascular Disease & Cerebrovascular Disease & $6(4.8 \%)$ & $10(1.7 \%)$ & 0.046 \\
\hline
\end{tabular}

COPD: chronic obstructive pulmonary disease, CRF: chronic renal failure, MAP: mean arterial pressure

* Having at least one additional disease in his / her medical history

Table 3: ROC Analysis Result by In-Hospital Mortality Status

\begin{tabular}{llllll}
\hline & & D-Dimer & Fibrinogen & Albumin & DAR \\
\hline In-Hospital Mortality & AUC (95\% CI) & $0.757(0.716-0.797)$ & $0.637(0.586-0.689)$ & $0.766(0.724-0.809)$ & $0.773(0.734-0.812$ \\
& Cut-off level & $>292.5 \mathrm{ng} / \mathrm{mL}$ & $>423 \mathrm{mg} / \mathrm{dL}$ & $<4.015 \mathrm{~g} / \mathrm{dL}$ & $>56.36$ \\
& Sensitivity & $74.6 \%$ & $68.3 \%$ & $81 \%$ & $84.9 \%$ \\
& Specificity & $67.3 \%$ & $56.5 \%$ & $59.4 \%$ & $58.4 \%$ \\
& PPV & $32.8 \%$ & $25.1 \%$ & $29.8 \%$ & $30.3 \%$ \\
NPV & $92.6 \%$ & $89.3 \%$ & $93.6 \%$ & $94.8 \%$ \\
\hline
\end{tabular}

CI: Confidence interval; AUC: Area under the curve; PPV: Positive Predictive Value; NPV: Negative Predictive Value

Table 4: Odds ratio results obtained according to optimum cut off values

\begin{tabular}{llll}
\hline & & Odds rate & $95 \%$ CI \\
\hline in-Hospital Mortality & D-Dimer $>292.5 \mathrm{ng} / \mathrm{mL}$ & 6.058 & $3.915-9.372$ \\
& D-Dimer $<292.5 \mathrm{ng} / \mathrm{mL}$ & 1 & \\
& Fibrinogen $>423 \mathrm{mg} / \mathrm{dL}$ & 2.794 & $1.856-4.205$ \\
& Fibrinogen $<423 \mathrm{mg} / \mathrm{dL}$ & 1 & \\
& Albumin $<4.015 \mathrm{~g} / \mathrm{dL}$ & 6.216 & $3.869-9.985$ \\
& Albumin $>4.015 \mathrm{~g} / \mathrm{dL}$ & 1 & \\
& $\mathrm{DAR}>56.36$ & 7.898 & $4.721-13.213$ \\
& $\mathrm{DAR}<56.36$ & 1 & \\
& FAR $>112.33$ & 4.437 & $2.911-6.762$ \\
& FAR $>112.33$ & 1 & \\
& $\mathrm{DAR}>56.36$ and FAR $>112.33$ & 21.879 & $9.879-48.703$ \\
& $\mathrm{DAR}<56.36$ and FAR $<112.33$ & 1 & \\
\hline
\end{tabular}

CI: Confidence interval

Figure Legends 
Figure 1: ROC Curve by In-Hospital Mortality

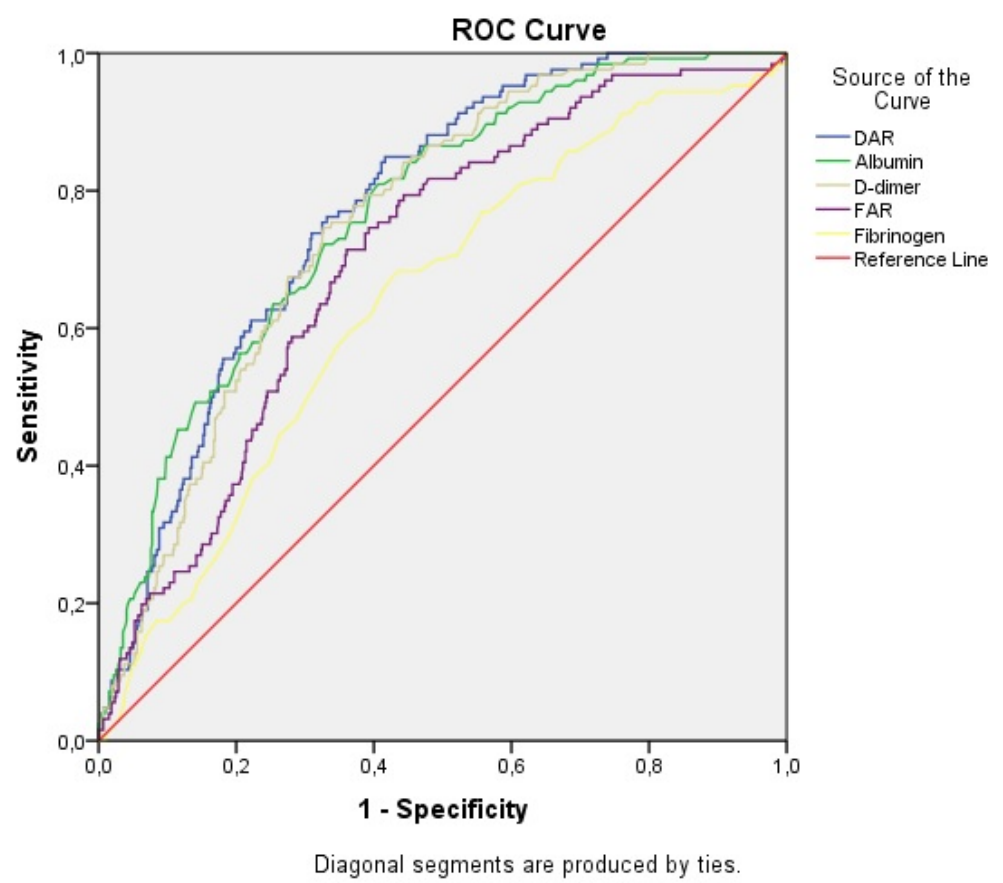

